\title{
A PSO Application in Skull Prosthesis Modelling by Superellipse
}

\author{
Marcelo Rudek ${ }^{*}$, Osiris Canciglieri Junior ${ }^{*}$ and Thiago Greboge ${ }^{*}$ \\ * Industrial and Systems Engineering Graduate Program - PPGEPS \\ Pontifical Catholic University of Parana - PUCPR \\ R. Imaculada Conceicao, 1155 - Curitiba-Parana-Brazil
}

Received $10^{\text {th }}$ Oct 2012; accepted $27^{\text {th }}$ Feb 2013

\begin{abstract}
This paper presents a method to create the geometric model of skull defects to be applied in anatomic prosthesis modelling. The approach is to generate an image that represents the missing information in the skull when bone's defect is non-symmetric. We are proposing the use of superellipse concept to recover the parameters that represents the geometric shape of a skull bone curvature in tomography. If the superellipse is properly adjusted in each computed tomography slice, the arcs that represent the piece of missing bone can be modelled in 3D. The problem is that many similar ellipses can be created, and the best solution must be found. This research applies the Particle Swarm Optimization (PSO) algorithm in order to find the best solution for each tomographic slice. Once the solution found for each slice, the whole 3D missing information can be virtually rebuilt as an adjusted prosthesis model image.
\end{abstract}

Key Words: Image Analysis, Prosthesis Design, Geometric Modelling, CAD, 3D Reconstruction.

\section{Introduction}

Nowadays, useful methods to surface reconstruction might be used to human body visualization, and the start point is the 3D reconstructed images from DICOM [1], [2]. Despite available data provided by medical images and integrated automated manufacturing resources, the handmade skull prosthesis is still reality in many surgical procedures [3], [4]. The quality requirements to surgical planning depend of doctor abilities and complexity of the defect.

We are proposing to use three-dimensional images obtained by tomography scans to generate the machining pre-processing parameters to automatically build anatomic skull prosthesis. In general the methods have focus about skull curvature, as [5] that have been proposed the approach called Bilaterally Symmetric Closed Fourier Curves based on the human body symmetry. In this case, we have some guaranties of the shape is very similar in the left or right side of human body as described by [6] [7]. This idea can be applied in the skull problems, because the existing good bone area of one side can be mirrored to repair a missing area in opposite side. However it is true only when the local of defect is symmetric from the both sides how shows [8]. In several cases, the position of skull defect does not permit use of symmetry information and traditional methods can't be applied. In this case, a missing bone piece image must be

Correspondence to: <marcelo.rudek@pucpr.br>

Recommended for acceptance by $<$ Georgios Triantafyllidis $>$

ELCVIA ISSN: $1577-5097$

Published by Computer Vision Center / Universitat Autonoma de Barcelona, Barcelona, Spain 
created from a criterion that guarantees their curvature. For this problem [9] proposed a method based on sub-cube pixel technique to obtain a surface model of a lost part of a defective skull. His work operates the 3D modelling by triangulations techniques.

Despite those good solutions presented, the problem addressed in this work is not only about 3D reconstruction and visualization to medical evaluation. We also need export data to a CAD/CAM (Computer Aided Design/Computer Aided Manufacturing) system thinking about a further step of machining. In this case, we need extract the necessary parameters embedded in the CT slices. Due some slices perimeter not completed because a hole, we need to generate additional information.

Due this reason, the study of [10] proposed a manner to adjust ellipses, slice by slice with parameters based on the skull border curvature to fill a missing area.

The image patterns that describe natural shapes can be represented by the superellipse concept. The [11] described its mathematical formulation and also create a superformula to be applied to plants and leaves shape identification. This theory also has been explored in many other ways, as show [12] and [13]. In our study, we are proposing to use this natural comportment in order to represent segments of human body as skull bone curvature. Depending of region of skull registered in the tomography, the circular form is different among all slices. So, the concept is adjusting ellipses to each one CT with different parameters. The problem is that several ellipses could be created with similar shape of a skull bone border for the same CT slice, differing themselves only by slight displacement in their parameters.

In engineering terms the necessity is to decide what the best solution might be found to proceeds an accurate machining operation. In this context, the evolutionary algorithms have been implemented successfully in several areas as well as image processing and shape recognition as [14][15][16].

The aim of the work is decide what ellipse represents the best adjust for each bone border in a specific CT slice. To generate the ellipse we are adjusting the superellipse parameters in order to estimate the data that represents it curvature. The parameters can be achieved through optimization methods. The PSO (Particle Swarm Optimization) can be used together with superformula as shown in [17] and together ellipses generation as demonstrated in [10]. It can be used in order to decide what is the best solution (best ellipse) that represents the bone curvature to each CT slice. The found ellipses parameters could be used in CAD profiles to generate the 3D machining model.

The contribution of work is about integration with machining that addresses the asymmetric case in skull repairing.

\section{Proposed Method}

By engineering viewpoint the prosthesis design process can be automated in some specific parts. The image correction is a necessary requirement in middle of process, among data patient acquisition and piece machining.

The proposed method to prosthesis modelling is stated as figure 1 .

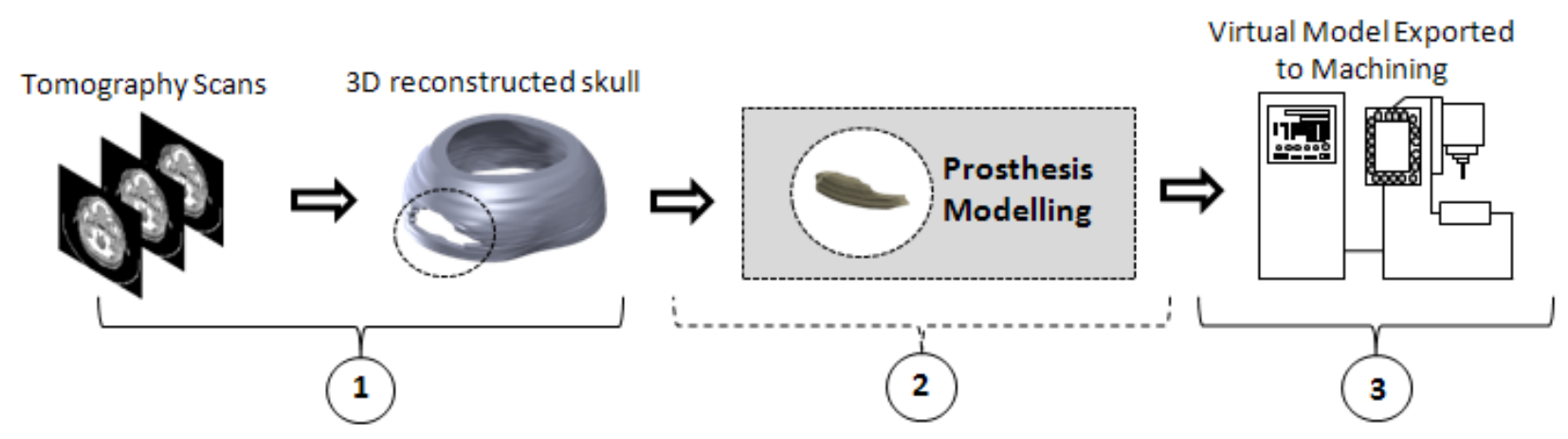

Figure 1. Diagram representing the methodology of the proposed process, adapted from [10]. 
The figure 1 shows the flow process arranged in three layers as:

i) The first level is the acquiring and conversion of the tomography image. This is the first step and consists in extracting the considering image portion from DICOM (Digital Image Communication in Medicine) file format. The information is achieved when the scanned sections pass through applied image segmentation algorithms to separation of brain and other tissues from bone to obtain only skull border information. Also, contains the method to $3 \mathrm{D}$ reconstruction and visualization of a virtual model of entire real skull. Here, the problem (hole in skull) is identified.

ii) The second level deals about the generation of the geometrical model of missing piece of bone. This layer is the main part of this research due handling of proposed optimization technique in each slice of $\mathrm{CT}$ followed by $3 \mathrm{D}$ reconstruction.

iii) The third layer is the final stage. The virtual model representation created in previous stage is prepared to CNC machining commands. This is a part that involves manufacturing requirements and it is not addressed here.

The interest part in this research is the second stage (layer 2) of the proposed process. The shape of skull bone has a circular form that seems an elliptical form in a CT slice. Despite the shape is not exactly an ellipse, an arc formed by an adjusted virtual ellipse can fill a gap in the bone for each CT image as shown in figure 2 .

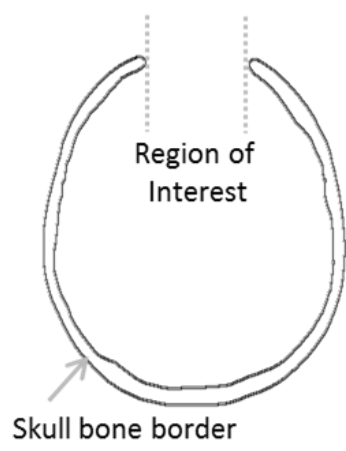

(a)

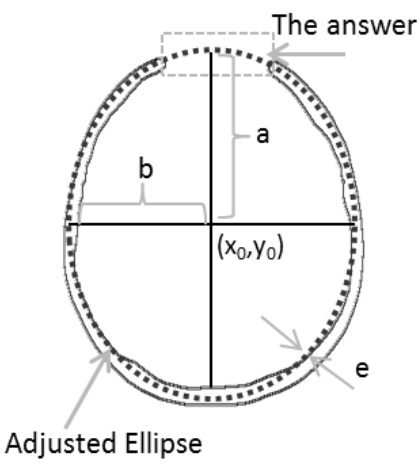

(b)

Figure 2. (a) Example of CT slice with a region to be fitted. (b) Ellipse parameters identification.

The figure 2.a shows an example of a CT slice with an uncompleted bone region. Figure 2.b shows the parameters to be find, as minor and major diameter $a$ and $b$, the centre $\left(x_{0}, y_{0}\right)$ of ellipse and $e$ is the thickness of the edge. Slight changing in the values of these parameters generates a lot of possibilities to create arcs and the more feasible answer must be finding. The aim is find the best ellipse that fits the skull border for each CT slice. The proposed method combines the formulation to generate an ellipse together with PSO optimization as described in sequence.

\subsection{Superellipse definition}

Due skull anatomy, the bone's curvature is different among all CT slices, and part of them seems ellipses (basically on frontal and parietal regions). The bone in each image can be modelled as ellipse with different shapes. To perform the ellipse generation, we are investigating the superellipse concept as presented by [11]. 
This concept permits the creation of geometric shapes as commonly found in nature. The mathematical formulation of the superellipse is presented in equation 1.

$$
r(\varphi)=\frac{1}{\sqrt[n_{1}]{\left(\left(\left|\frac{1}{a} \cos \left(\frac{m}{4} \varphi\right)\right|\right)^{n_{2}}+\left(\left|\frac{1}{b} \sin \left(\frac{m}{4} \varphi\right)\right|\right)^{n_{3}}\right)}}
$$

From equation (1) the parameters $a$ and $b$ represents the minor and major axis of ellipse. The $m$ value represents the rotational symmetry, which is the number of fixed arguments on the unitary circle. The obtained shape is a rounded polygon and $m$ describes the number of sides of this polygon. For example, for $m=3$ we obtain a triangular shape and for $m=0$ we obtain a circle. The values of $n_{2}$ and $n_{3}$ determine if the shape is inscribed or circumscribed in a unitary circle. The figure 3 shows some plot examples by modifying of superllipse parameters. Additional interesting examples applied together superformula can be seen in [11].

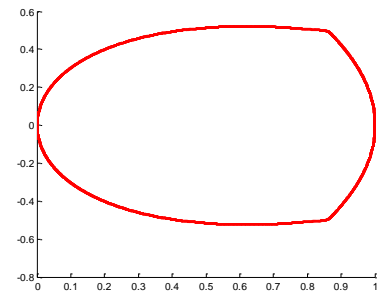

(a)

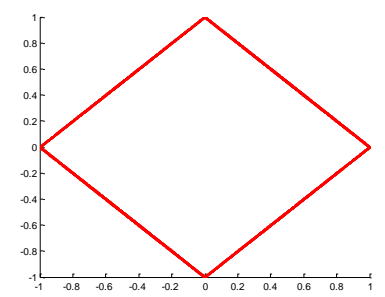

(c)

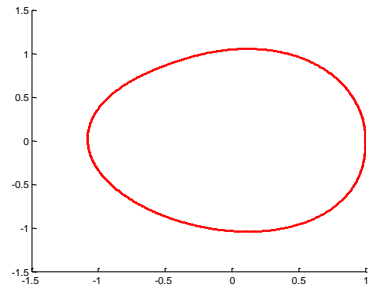

(b)

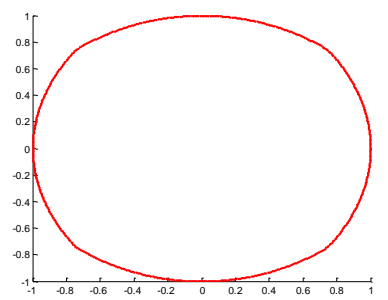

(d)

Figure 3. Examples of plots for different superellipse parameters. (a) $[a=1$, $b=1, m=6, n 1=250, n 2=100, n 3=100] ;$ (b) $[a=1, b=1, m=3, n 1=2, n 2=3, n 3=2] ;$ (c) $[a=1, b=1, m=4, n 1=1, n 2=1, n 3=1]$; (d) $[a=1, b=1, m=4, n 1=200, n 2=30, n 3=30]$.

In figure 3 is observed different polygon's achieved by modified values of superellipse parameters. The polygon in figure 3.a has six fixed points $(m=6)$ and figure 3.b has three fixed points $(m=3)$ as a triangle, but in these cases with rounded border due modified $n$ values. The figure 3.c and 3.d have the same $m$ value, but $n$ determines the curvature that not appears in figure 3.c due unitary values. An ellipse can be obtained if $n_{1}=n_{2}=n_{3}=2$ and $m=4$, and in this case we have the traditional ellipses' equation. By observation, some slices in the middle of skull seem as a normal ellipse (by the equality of parameters $n$ ). When the shape turns more oval, on the skull top for instance, the superellipse can self-adjust better than the normal ellipse by adjusting the values of $n$.

To plot the shape, we can consider the $E(i, j)$ as the binary value of a pixel in the current position $(i, j)$ in generated ellipse. The ellipse point $\mathrm{E}(\mathrm{i}, \mathrm{j})$ can be obtained by polar coordinates, as presented in equation 2 .

$$
E(i, j)=\left(r * \cos \varphi+x_{0}, r * \operatorname{sen} \varphi+y_{0}\right)
$$

We are adapting this concept to generate elliptical shape to self-adjustment in skull border, as in figure 4 . 
The ellipse found by superellipse formula as the example in figure 4.a, is one whose parameters represents a possible curvature around bone (in this example only represented the external border). But, is expected which there are slight differences between generated ellipse and real bone shape because the bone perimeter is not a perfect circle.

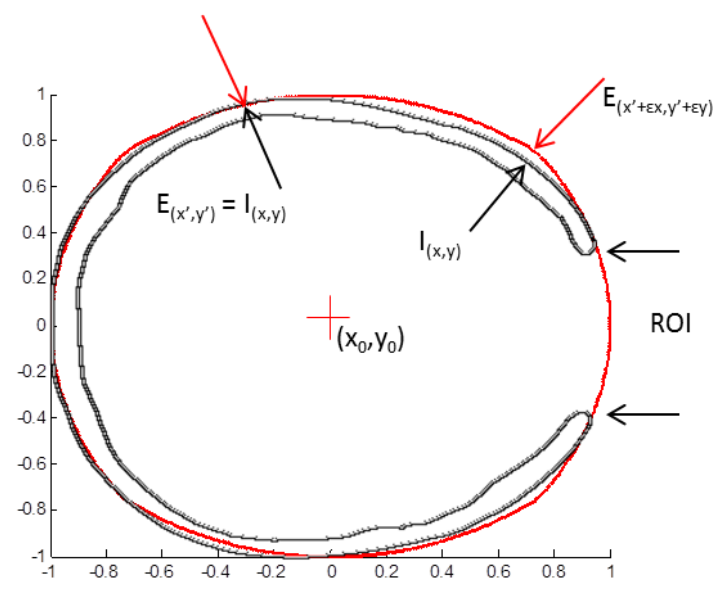

(a)

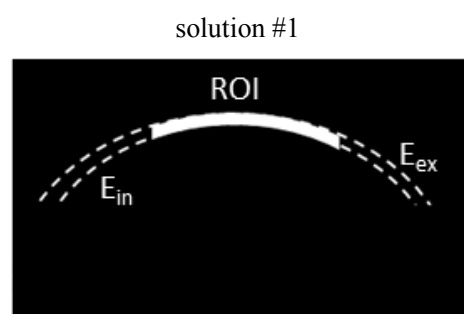

(b)

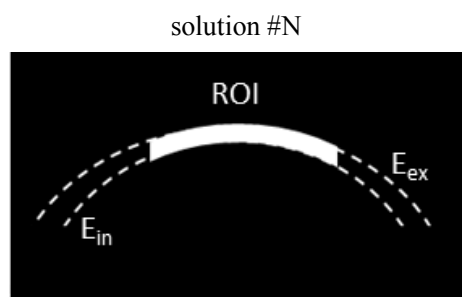

(c)

Figure 4. (a) An example of superllipse adjustment overlapped in external skull border. (b) A sketch of one possible adjustment to external $\left(E_{\text {ex }}\right)$ and inner $\left(E_{\text {in }}\right)$ borders. (c) A sketch of a different possibility to ROI filled with different ellipse $E_{\text {ex }}$ and $E_{\text {in }}$ parameters.

In this approach, the main problem is that many arcs can be found with similar information to the same CT slice to external and inner borders. It can be seen in figures 4.b and 4.c the different results in ROI (Region of Interest) area. Among a lot of possible arcs created for each slice, the question here is to decide what the best solution is. The decision is about what superllipse parameters will permit to create the best piece of curved shape for this uncompleted bone. The search about these parameters might be optimized to find the best set of them.

\subsection{Particle Swarm Optimization (PSO)}

The PSO (Particle Swarm Optimization) has proven to be efficient in solving optimization problem [18][19]. As presented by [20] in PSO, each possible solution is called particle and it is a part of the swarm (population). Each particle is initialized with a random position value and after, flies around the multidimensional search space and adjust its position based on its own experience and also on its neighbors' experience.

The fitness function $F(X)$ to evaluate the ellipse adjustment was proposed in [10] and it is given by equation 3. The image $\mathrm{I}$ is a black \& white segmented CT slice. Each point $\mathrm{I}(i, j)$ is the position of one pixel in the image where its binary value is 1 . The $l$ and $c$ values are respectively the total of lines and columns in the image I.

$$
F(X)=\frac{\sum_{i=1}^{l} \sum_{j=1}^{c}[I(i, j) * E(i, j)]}{\sum_{i=1}^{l} \sum_{j=1}^{c} I(i, j)}
$$

The $r$ value is the generated parameter in equation 1 to estimate the $\mathrm{E}(x, y)$ pixel position by evaluation of equation 2 . The values of $a, b, m, n_{1}, n_{2}, n_{3}$ from equation 1 , centre coordinates $x_{0}, y_{0}$ and thickness $e$ are all 
estimated by the PSO algorithm. During the simulation, each iteration the fitness function is evaluated using the current position of the particle using, the personal best value, namely pbest, and, global best value, namely gbest. The personal best value is the location of the best value obtained so far by the particle. The global best value is the location of the value obtained so far considering all the particles in the swarm, as the follow pseudo code adapted from [14].

\section{Initialize particle:}

A vector with ellipse parameters $a, b, x_{0}, y_{0}, m, n_{1}, n_{2}, n_{3}$ is randomly assigned among a range of possible values. The possible thickness values $e=1, \ldots, 8$.

\section{Evaluate particles:}

Do

For each particle:

- Calculate the fitness value $F(x)$ by equation 3.

- If $F(x)$ is better than actual pbest set the current values as pbest.

End

For each particle:

- Find in the neighbourhood the particle with the best fitness among all. Set it as gbest.

- Calculate particle velocity according to the velocity equation*.

- Update particle position according to the position equation*.

End

While (maximum interactions) not obtained.

*The complete definition about 'velocity' and 'position' equations can be obtained from [20].

The both bone borders from external and internal skull walls must be evaluated. The maximum and minimum values of $a$ and $b$ parameters must be limited to avoid that one overtake other. Also, the changes in thickness (parameter $e$ ) can perform a weak or more precise adjust. A case study was select to demonstration the optimization process.

\section{Case Study}

A case study simulating a hypothetical condition of a missing area of the bone of a human skull was used to evaluate the method. The figure 5 shows the $3 \mathrm{D}$ skull image. The hole in the bone was virtually made slice by slice as in figure 5.a. The figure 5.b shows the hole's allocation addressed in the example.

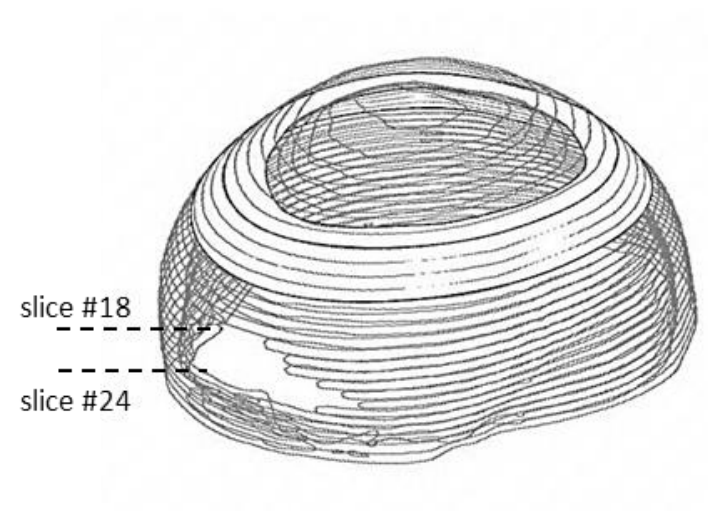

(a)

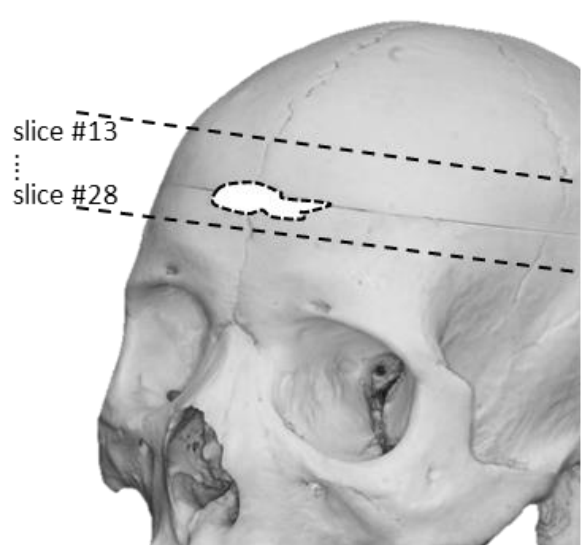

(b)

Figure 5. (a) 3D reconstructed skull with respective defect. (b) Selected skull region. 
A partial area with a hole in frontal region of skull was used to simulate a defect to an asymmetric case (no symmetry between left and right sides of skull). A range with sixteen slices numbered as indicated in figure 5.b was used to demonstrate the method`s application. The slices \#18 till \#24 represents the hole`s height. Figure 6 shows some samples of adjustments using the selected slice. In each figure can be seen the bone's border with its respective ellipse superposed. The slice number \#20 was used to demonstration of adjustment presented in figure 6.a.

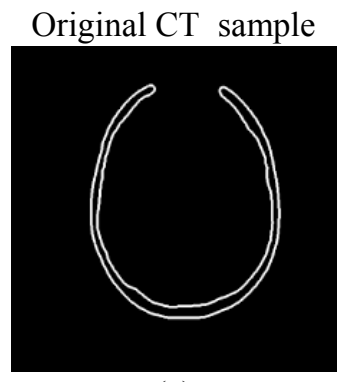

(a)

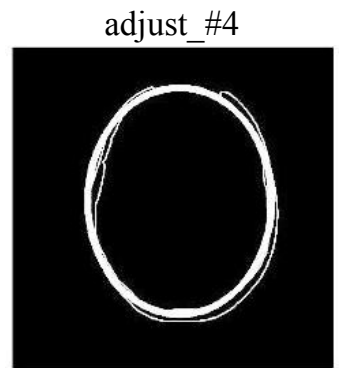

(e)

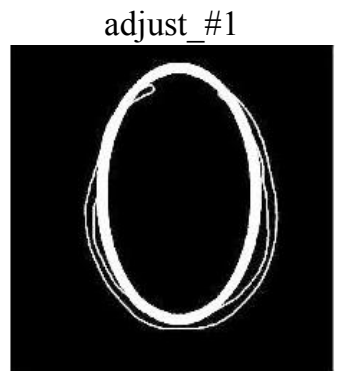

(b)

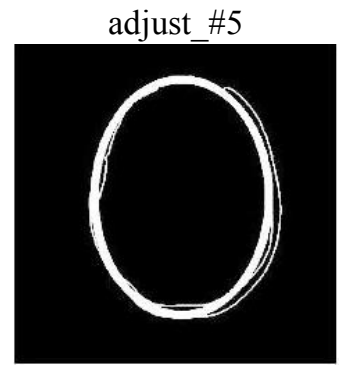

(f)

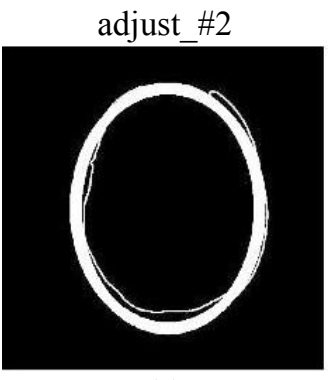

(c)

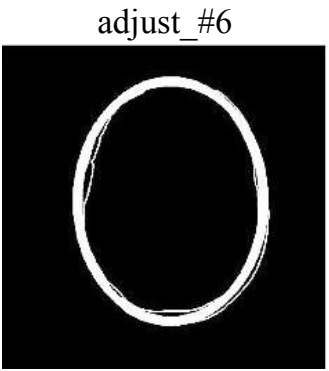

(g)

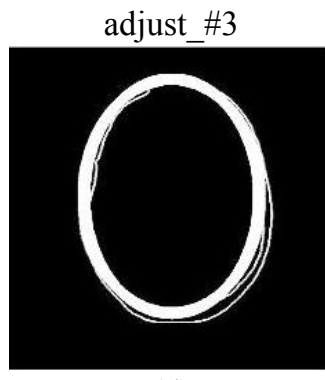

(d)

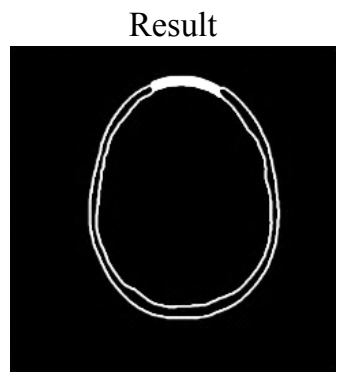

(h)

Figure 6. CT slices and samples of ellipse adjustments.

The parameters generated by PSO permits to draw different ellipses as shown in figures $6 . \mathrm{b}$ to $6 . \mathrm{g}$ to the same CT slice. Each drawn ellipse was created based on optimized values generated from PSO algorithm, and we need choose the best one. To the analysed region (slices between number \#18 and \#24) the superellipse parameters was adjusted as a normal ellipse with $n_{1}=n_{2}=n_{3}=2$ and $m=4$. Table 1 presents a representative set of virtual ellipses fitness values and its respective parameters with a range of six possibilities to the same CT slice (number \#20) as example.

\begin{tabular}{c|c|c|c|c|c|c}
\hline adjust & $\boldsymbol{x}_{\boldsymbol{0}}$ & $\boldsymbol{y}_{\boldsymbol{0}}$ & $\boldsymbol{a}$ & $\boldsymbol{b}$ & $\boldsymbol{e}$ & Fitness (\%) \\
\hline$\# 1$ & 189 & 145 & 257 & 267 & 8 & 29,2898 \\
$\# 2$ & 192 & 122 & 232 & 269 & 7 & 51,0782 \\
$\# 3$ & 185 & 138 & 238 & 266 & 8 & 54,8275 \\
$\# 4$ & 180 & 146 & 247 & 268 & 4 & 51,1811 \\
$\# 5$ & 187 & 138 & 247 & 275 & 5 & 60,0475 \\
$\# 6$ & 188 & 146 & 247 & 270 & 6 & 61,5324 \\
\hline
\end{tabular}

Table1. Optimized parameters from a same sampled CT.

Among all ellipses generated for the same slice, the fitness value help us to decide what the best adjustment is. The adjustment numbered as $\# 6$ is the found solution because it has the better ellipse parameters. This resulting ellipse is superposed on bone edge, and by subtraction it is possible to separate only the arc that fits that missing area. The final adjustment can be seen in figure 6.h. 


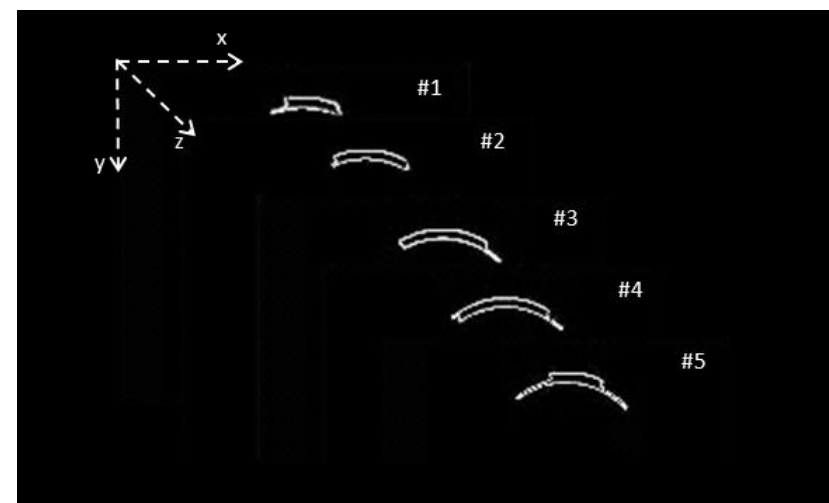

(a)

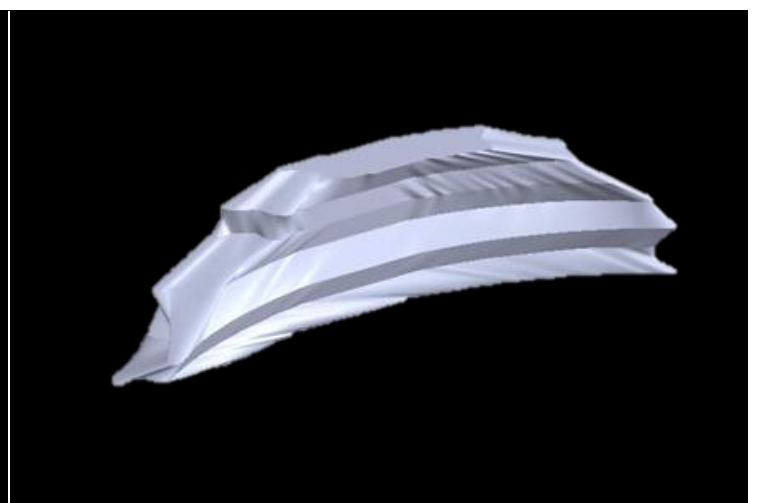

(b)

Figure 7. (a) Sample of pieces found to each remain slice. (b) Reconstructed model.

The same evaluation process is done for all other remained slices, and, for each one we can use the individual solution found to build a 3D reconstructed piece. The figure 7 shows the individual solutions for a sampled set of CT slices. They are used to create virtual 3D piece prosthesis. The individual solution for each CT slice was superposed to build a virtual 3D prosthesis model as presented in figure 7.a. The figure 7.b shows the completed reconstructed structure that represents the missing bone region.

The numeric result generated by proposed method is presented in table 2 . The fitness values for a set of six $\left(e_{1}, e_{2}, e_{3}, e_{4}, e_{5}, e_{6}\right)$ sampled slices are shown as example. Based on those values, an example with minor set of ellipses was drawn to illustrate the arc positions and respective surface as indicated in figure 8 , where each $e_{n}$ ellipse represent its respective $\mathrm{CT}$.

\begin{tabular}{l|rrrrrr}
\hline \multicolumn{1}{c|}{ PSO } & \multicolumn{6}{c}{ Generated Ellipse } \\
\multicolumn{1}{c}{ Algorithm } & \multicolumn{1}{c}{$\mathrm{e}_{1}$} & \multicolumn{1}{c}{$\mathrm{e}_{2}$} & \multicolumn{1}{c}{$\mathrm{e}_{3}$} & \multicolumn{1}{c}{$\mathrm{e}_{4}$} & \multicolumn{1}{c}{$\mathrm{e}_{5}$} & \multicolumn{1}{c}{$\mathrm{e}_{6}$} \\
\hline Best Fitness (\%) & 73.6621 & 69.6184 & 53.0127 & 69.5255 & 69.5981 & 64.5173 \\
Worst Fitness (\%) & 42.3930 & 61.3342 & 51.5145 & 31.0352 & 56.8046 & 36.8291 \\
Mean Function (\%) & 69.5516 & 67.7773 & 52.2834 & 56.1286 & 67.9126 & 51.2728 \\
Median Func. (\%) & 73.6621 & 68.6799 & 52.0166 & 62.1287 & 68.8226 & 54.4475 \\
Standard Deviation & 0.0022 & 0.0005 & 0.0001 & 0.0042 & 0.0007 & 0.0037 \\
Medium Time (s) & 184.137 & 176.457 & 173.954 & 177.937 & 174.888 & 177.915 \\
\hline
\end{tabular}

Table 2. Fitness values to six sampled CT slices.

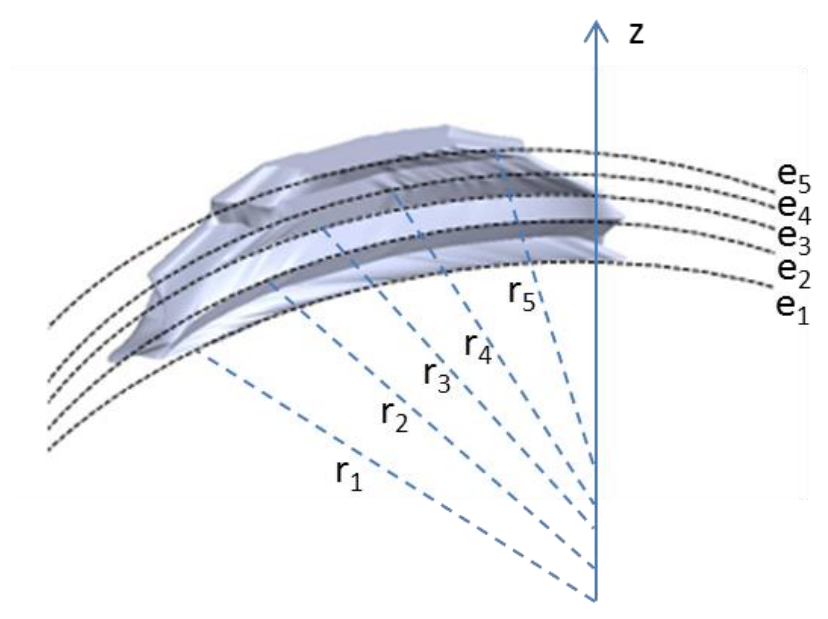

Figure 8. 3D piece of virtual model by superposed arcs from each CT slice. 
The figure 8 shows the inner side of constructed model with respective distances $r_{i}$ from centre to each generated ellipse $e_{i}$, with $i=1, \ldots n$ possibilities. Without losing the generality we can consider each radius measurement $r$ as the same $a$ parameter from superellipse equation. Those all values that were found can be exported to CAD system to build the representative graph of obtained ellipse. The virtual model in CAD will permit define the parameters to planning in advance the machining process.

\section{$4 \quad$ Results and Discussion}

The experimental results were obtained from the proposed study case, based in an amount of 16 CT slices in the region of interest (CT \#13 to CT \#28) as indicated previously in figure 5. Among those CT we have 9 slices with closed borders and 7 with open contours. Through the specific characteristics from presented problem, by observation of image data, we were able to define the initial set of parameters to start the PSO algorithm. The initial parameters were initialized with a limited values set where the values of axis $a$ and $b$ have a variation about 15 and 10 pixels respectively from original centre of slice. For instance, taking as base the best fitness values found in the simulations, the range to length of axis $a$ is between $\min =229$ and $\max =259$, and the range of the axis $\mathrm{b}$ length is between $\min =261$ and $\max =281$. The position of the centre, the coordinate $\mathrm{x}_{0}$ has a range between $\min =180$ and $\max =192$, and the coordinate $\mathrm{y}_{0}$ has a range between $\min =122$ and $\max =146$. The variance to position of the ellipse centre in absolute values is 6 pixels to $\mathrm{x}_{0}$ and 12 pixels to $\mathrm{y}_{0}$.

The figure 9 shows the generated ellipses in a CAD system according parameters obtained from best fitness adjustment for each tested CT slice. The number of iterations of PSO algorithm was 100 for each slice. Figure 9.a show the ellipses that represents the internal edge of bone, and figure 9.b presents the ellipses adjusted to external skull border. The difference of the centre positions and axis length can be seen in the both results. Based on evaluation of comportment of mean, median and deviation in this study case, the number total of iterations could be reduced to more than a half part because the fitness value that maximize the function $F(x)$ is found around 20 to 30 iterations for each slice. This generates important reduction of processing time.

Also, depending if ellipse adjustment occurs on open or closed border slices, the fitness values indicates what border type is. If fitness is around $60 \%$ to $80 \%$ we know that it represents an adjustment of an open border, and if fitness values are more than $80 \%$ is because the border is closed. This is occurs because in a closed border there are a major amount of coincident pixels present between in $E(\mathrm{i}, \mathrm{j})$ and $I(\mathrm{i}, \mathrm{j})$ in equation 3 . This is an observed fact, but complementary analysis is still necessary to prove whether this interpretation can be generalized for different cases.

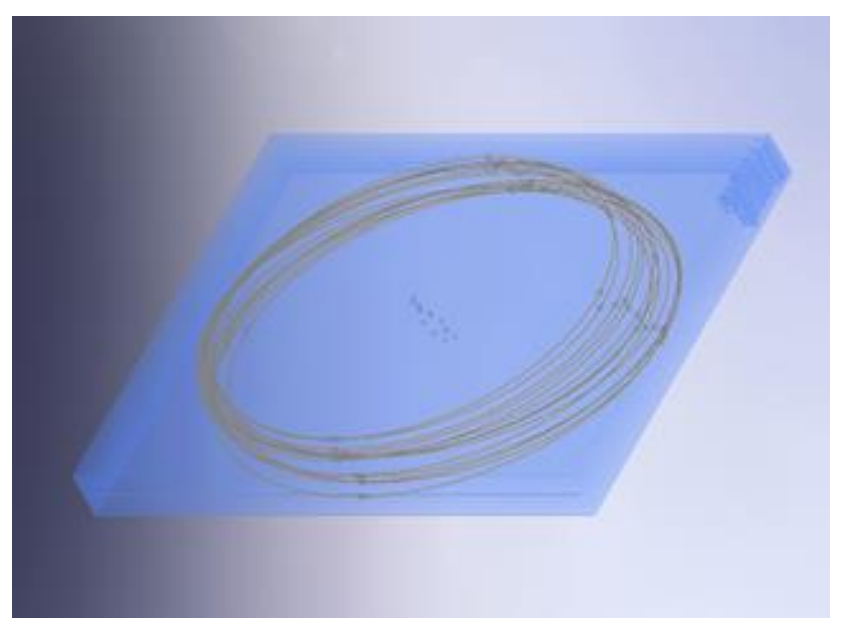

(a)

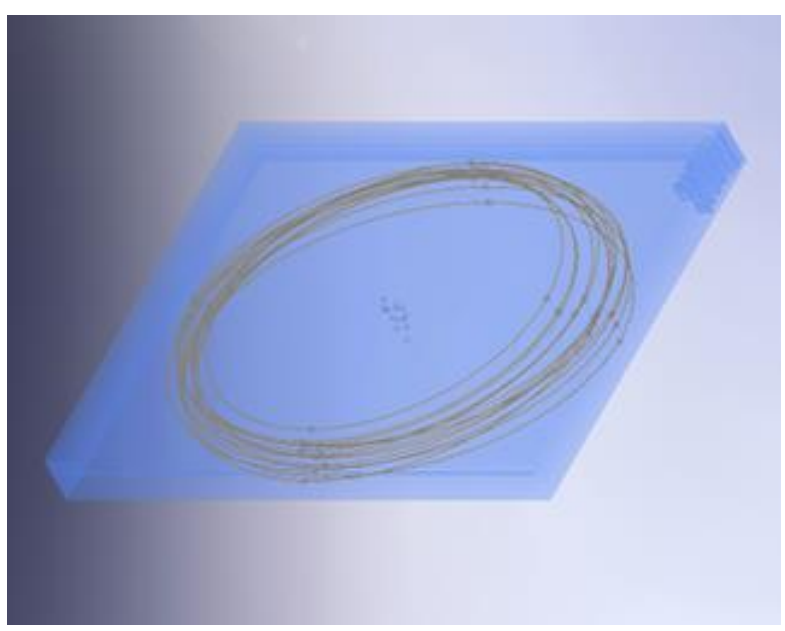

(b)

Figure 9. (a) The 3D skull reconstruction in CAD. (b) Prosthesis model 
The ellipse parameters of all generated curves were used as input in CAD software. An ASCII text file with tabled values is imported by the CAD system, and it made the interpolation by an automatic way to create the surface and its $3 \mathrm{D}$ visualization. In this example, the curves $e_{i}$ were equally spaced because the purpose was also to evaluate the interpolation accuracy to surface generation. The best fitness values (as shown in table 2) were used to get the ellipse parameters in order to generate the prosthesis adjusted in the skull as indicated in figure 10 .

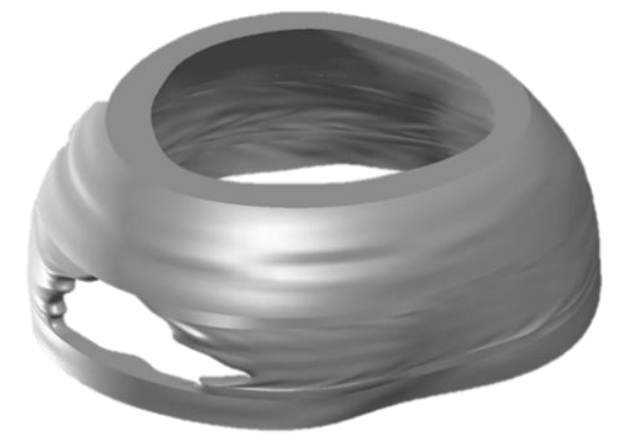

(a)

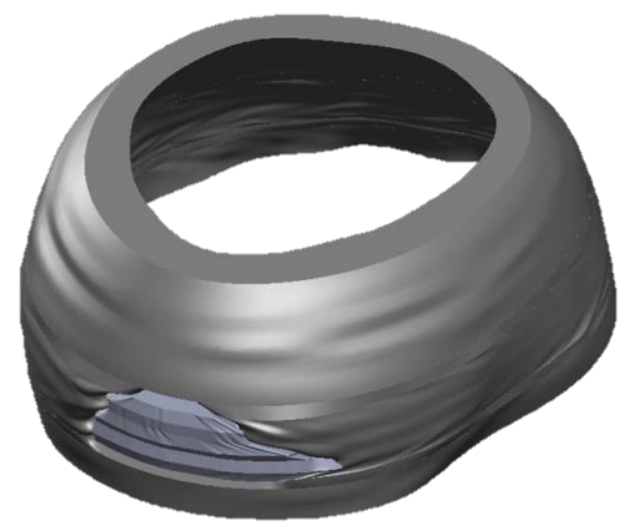

(c)

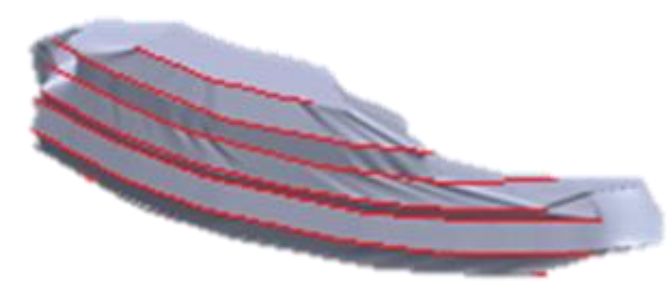

(b)

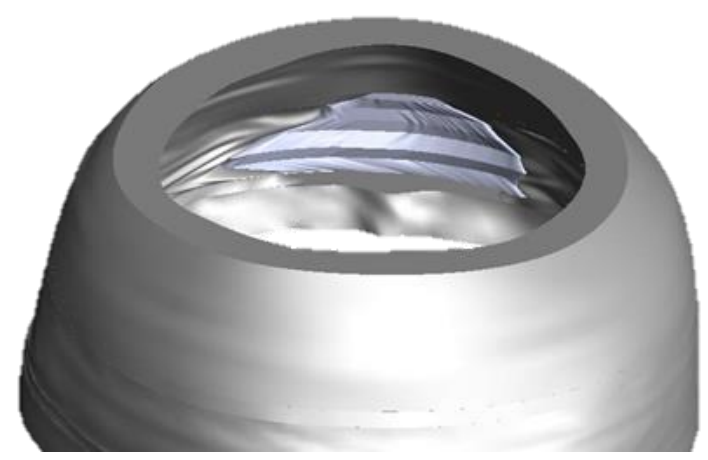

(d)

Figure 10. (a) The 3D skull reconstruction in CAD. (b) Prosthesis model with respective ellipses.

(c) Adjusted virtual prosthesis of outer side view. (d) Adjusted prosthesis of inner side view.

In figure 10.a we have the original testing image of a skull with respective hole reconstructed in CAD. In figure 10.b it is presented the 3D model of calculated prosthesis that fills the hole in the skull and respective external arcs to each tested CT slice. It is possible to see in the surface, an interpolation problem between each arc. The apparent distortion is a problem to be explored in a future work. The figures 10.c and 10.d are two views of reconstructed prosthesis model that fits the hole in the external and internal bone's wall of skull. Through a visual inspection we can observe that it is possible to generate a curved surface as skull shape using the proposed approach by ellipse. Despite this observation the method still presents some problems and due this, the model is not feasible to real application yet. For instance, there are some problems such as the union between the real bone segment and the reconstructed piece have appeared in some cases. The figure 11 shows an example of junction problem.

This problem is highlighted in 3D reconstructed piece as in figures 10.c and 10.d where the shadows and lights positions emphasize a discontinuous link between prosthesis and bone. Virtual illumination from software causes a "rounded effect" in extremities and it is not desirable in this case because it might cause a confused visualization. This is still an open question that will not be addressed now. 


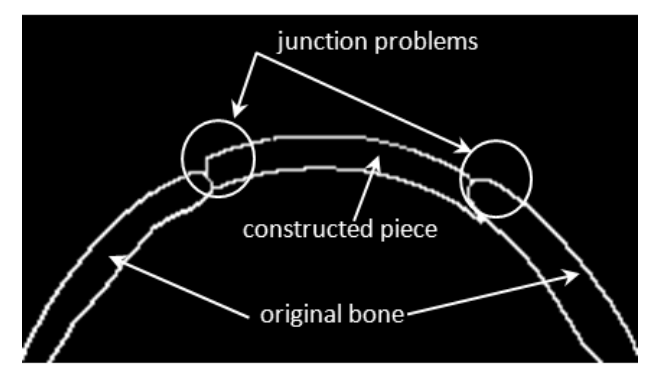

Figure 11. Non-accurate junction after ellipse`s adjustment.

Despite some adjustment problems are visible in 3D, the conceptual idea about creation of a virtual model to machining a piece from C.T. images appear as an possible way to generate information when we don't have symmetry and when a mirroring process can't be used.

Other methods as Genetic Algorithm (GA) and Harmony Search (HS) discussed in [14] are still under study. Nevertheless PSO has been demonstrated upper among them for the cases studied. The next step in the research is CAM preparing in order to machining.

\section{Conclusion}

This paper presented a method to prosthesis modelling, based in the concept of ellipses that were capable to perform a self-adjustment at bone curvature through optimization techniques called PSO. The case study used to validate the method, established that it is possible to build a virtual model of part of bone whose information do not exists in the image (without symmetry). Although this information can be obtained by other means such as numerical methods or user interaction, the question is how to find a way of modelling the prosthesis automatically.

The accuracy is a fundamental requirement to turn the method in a functional tool. Moreover, other optimization methods based on artificial intelligence might be used to improve the ellipse adjustment and are being explored in order to continue the research. It is, also necessary future steps of study to answer the points that still open and which are:

- The analysis about the quality of the results based on a set of images of a skull with real fractures or anatomical defects;

- The evaluation of the measurement accuracy between the 3D reconstructed image and the real bone considering the prosthesis fixation.

- The medical analysis viewpoint about the functional aspects of the method, to review surgery planning and about the procedures in the implant methods;

- The machining of prosthesis using CAD/CAM technology and the comparison of the real skull shape for both structures - the skull and prosthesis prototyping;

Despite some question are still open, the union of image processing tools and optimization techniques open an important range of possibilities to complete this research.

\section{References}

[1] W. D. Bidgood, S. C. Horil, F. W. Prior, D. E. Van Syckle, Understanding and using DICOM, the data interchange standard for biomedical imaging, J. Am. Med. Inform. Assoc., v.4, 199-212, 1997.

[2] (DICOM) Digital Imaging and Communications in Medicine Part 5: Data Structures and Encoding, National Electrical Manufacturers Association, 17th Street Rosslyn, Virginia 22209 USA, 2011. 
[3] G. Fatterpekar, A. H. Doshi, M. Dugar, B. Delman, T. Naidich, P. Som. Role of 3D CT in the evaluation of the temporal bone. Department of Radiology, Mount Sinai Medical Center, New York, USA, 2006.

[4] B. C. Brevi, A. S. Magri, L. Toma, E. Sesenna, Cranioplasty for repair of large bone defect with autologous and homologous bone in children, Journal of Pediatric Surgery, v. 45, 17-20, 2010.

[5] H. Li ; Y. Pei ; J. Zhao ; L. Wu ; Design and manufacturing of titanium alloy cranial prosthesis based on forward and reverse principle. 2010 International Conference on Mechanic Automation and Control Engineering (MACE, 5710 - 5713), 2010.

[6] D. Sengupta, P. Ghosh, Bilaterally Symmetric Fourier Approximations of the Skull Outlines of Temnospondyl Amphibians and their Bearing on Shape Comparison. Journal of Biocience. 30:3:377390, 2005.

[7] H. Li, Z. Xie, S. Ruan, H. Wang, "The Measurement and Analyses of Symmetry Characteristic of Human Skull Based on CT images". Biomechanics and Vehicle Safety Engineering Centre, Tianjin University of Science and Technology, Tianjin, China, Vol. 26, No. 1, 34-37, 2009.

[8] T. Greboge, O. Canciglieri Jr,, M. Rudek, "Modelagem 3D de Próteses Baseada em Imagens de Tomografia Computadorizada", XVII Simpósio de Engenharia de Produção - SIMPEP, Bauru, SP, Brazil, 2010 (in Portuguese).

[9] F. You.; Q. Hu; Y. Yao; Q. Lu, "A New Modeling Method on Skull Defect Repair", IEEE International Conference on Measuring Technology and Mechatronics Automation, 2009.

[10] M. Rudek, O. Canciglieri Jr., T. Greboge. An Optimized Method for Anathomic Skull Prosthesis Modelling. 13Th IASTED Conference in Computer Graphics and Image, CGIM2012, Crete-Greece, 2012.

[11] J. Gielis, A Generic Geometric Transformation That Unifies a Wide Range of Natural and Abstract Shapes, American Journal of Botany, v.90, p.333-338, 2003.

[12] M. Spehr, S. Gumhold, R. W. Fleming, Sum-of-Superellipses - A low parameter model for amplitude spectra of natural images. 2011.

[13] X. Zhang, P. L. Rosin. Superellipse fitting to partial data. Pattern Recognition, v. 36, 2003, p.743-752.

[14] T. Greboge; R. B. Grebogi; M. Rudek; O. Canciglieri Jr.. Geometric Prosthesis Modeling to Skull Repairing Using Artificial Intelligence Methods. In: 41st International Conference on Computers \& Industrial Engineering (CIE 41), Los Angeles, 2011.

[15] S Hashemi, S. Kiani, N. Noroozi, M. Moghaddam, "An Image Enhancement Method Based on Genetic Algorithm", IEEE International Conference on Digital Image Processing, New York, NY, USA; 167-171, 2009.

[16] R. Jennane, R., Almhdie-Imjabber, A., Hambli, R., Ucan, O.N., Benhamou, C.L., "Genetic Algorithm and Image Processing for Osteoporosis Diagnosis", IEEE Annual International Conference of Engineering in Medicine and Biology, Buenos, Aires, Argentina, pp. 5597-5600, 2010.

[17] S.K. Mishra, Some Experiments on Fitting of Gielis Curves by Simulated Annealing and Particle Swarm Methods of Global Optimization. Dept. of Economics NEHU, Shillong (India), 2007

[18] Y. Jiang, T. Hu, C. Huang, X. Wu. An improved particle swarm optimization algorithm. Applied Mathematics and Computation. v.193, p. 231-239, 2007.

[19] L. Chuang, S. Tsai, C. Yang. Chaotic catfish particle swarm optimization for solving numerical optimization problems. Applied Mathematics and Computation. v. 217, 6900-6916, 2011.

[20] J. Kennedy, R. C. Eberhart. Particle swarm optimization. In: Proceedings of IEEE International Conference on Neural Networks. Perth. v. 4, p. 1942-1948, 1995.

[21] M. Rudek; O. Canciglieri Jr;; T. Greboge. A Prosthesis Design Based on Genetic Algorithms in the Concurrent Engineering Context. In: ISPE Concurrent Engineering 2011 - CE2011, Boston. v. 1, 1224, 2011. 